\title{
A thermodynamic closure for the simulation of multiphase reactive flows \\ P. Boivin ${ }^{1 a}$, M.A. Cannac ${ }^{\mathrm{a}}$, O. Le Métayer ${ }^{\mathrm{b}}$ \\ ${ }^{a}$ Aix Marseille Univ, CNRS, Centrale Marseille, M2P2, Marseille, France \\ ${ }^{b}$ Aix Marseille Univ, CNRS, IUSTI, Marseille, France
}

\begin{abstract}
A simple thermodynamic closure for the simulation of multiphase reactive flows is presented. It combines a fully explicit thermodynamic closure appropriate for weakly thermal multiphase flow simulations, with the classical variable heat capacity ideal gas thermodynamic closure, commonly used for reactive flows simulations. Each liquid and gas component is assumed to follow the recent Noble-Abel Stiffened Gas equation of state, fully described by a set of five parameters. A new method for setting these parameters is presented and validated through comparisons with NIST references. Comparisons with a well-known cubic equation of state, Soave-Redlich-Kwong, are also included. The Noble-Abel Stiffened-Gas equation of state is then extended as to cope with variable heat capacity, to make the mixture thermodynamic closure appropriate for multiphase reactive flows.

Keywords: thermodynamics, equation of state, multiphase flows, reactive flows, computational fluid dynamics
\end{abstract}

\section{Introduction}

\footnotetext{
${ }^{1}$ Corresponding author: pierre.boivin@univ-amu.fr
} 
Fluid mechanics are governed by mass, momentum, and energy conservation equations. Any attempt at solving them requires consideration of a so-called thermodynamic closure to account for the pressure work, by providing the relation between pressure, mass and energy.

For compressible flows, implementation of such relation in a solver is nontrivial [1], as large pressure ratios are encountered. Additional difficulties are encountered when the flow is also multiphase [2, 3], with large density ratios being now encountered. When exothermic reactions also require consideration - e.g. for a burning liquid jet - the temperature also exhibits large ratios, and implementing an appropriate closure is challenging [4 6 ]. Within that last category, one of the various variants of the cubic Van der Waals equation of state [7-10] is often adopted [4 6$]$, for they are relatively simple and valid over large density-pressure-temperature ranges.

In subcritical conditions, however, the liquid and gas phases have such different thermodynamic properties that it can be easier to use separate equations. In that direction, Stiffened Gas (SG) EOS are a common option: they include very few independent parameters and can easily be tuned to well describe conditions of interest [11]. The Noble-Abel Stiffened-Gas (NASG) EOS is an improved version of the SG EOS proposed recently [12]. The authors' strategy for setting the equation of state parameters is, as in their previous work in the SG EOS context [11], based on the sole fitting of the saturation properties in terms of temperature, pressure, density and enthalpy. The approach is reasonable for multiphase flows remaining close to the saturation conditions, but this study shows that significant errors are obtained as soon as the flow departs significantly from the saturation properties. This will be 
especially overriding in multiphase reactive flows, where it is important to accurately assess the energy necessary to heat a product over wide ranges of temperatures. This work's objective is to offer an alternative method, allowing a good asymptotic representation in the pure liquid and vapor limits of the fluid.

The paper is organized as follows. First, the NASG EOS main features

will be recalled [12] along with its advantages over the SG description. The next section of the paper will describe a new method for setting the NASG EOS parameters. Validation of the new parameters will be shown, by comparing with the original work [11, 12] as well as the well known SRK EOS [9]. In the following section, the NASG EOS is extended as to cope with variable heat capacity. This extension finds its importance for applications that are both multiphase and reactive. Associated to the last section is an extension of the thermodynamic closure for two-phase flows consisting of a liquid and a multicomponent gas, all assumed to follow the NASG EOS (or associated stiffened or ideal gas simplifications).

\section{The Noble-Abel Stiffened-Gas Equation of State}

The NASG EOS for each phase reads:

$$
p(\rho, T)=\frac{\rho(\gamma-1) C_{v} T}{(1-\rho b)}-p_{\infty}
$$

where the usual notations are used. $p$ is the pressure, $\rho$ the density, $\gamma$ the heat capacity ratio, $C_{v}$ the mass heat capacity at constant volume (or at constant pressure, $\left.C_{p}=\gamma C_{v}\right), b$ the covolume, and $p_{\infty}$ is another parameter representing the molecular attraction in the liquid phase. Following [12], the coefficients $\gamma, C_{v}, b$ and $p_{\infty}$ are assumed to be constants. 
Equation (1) will be used for the liquid phase, whereas for the gas phase $b$ and $p_{\infty}$ are set to zero, reducing to a perfect gas description.

Figure 1 compares the density and enthalpy as obtained at constant pressure with the NASG EOS [12], the SRK EOS, and the reference from the NIST database for water $\mathrm{H}_{2} \mathrm{O}$. A clear disadvantage of the SRK EOS is that it leads to errors in liquid density on the order of $20 \%$, with the latent heat of vaporization overestimated by $5 \%$. Because the liquid and gas phases have separate EOS in the SG and NASG framework, it is easier to obtain more accurate thermodynamic properties in both phases.

One of the advantages of the NASG EOS over the SG EOS [12] is that it allows a much better description of the liquid phase. This is made possible by the introduction of the covolume $b$, which allows to lift the following limitations of the SG framework:

- $\rho$ is not no longer inversely proportional to $T$ at constant pressure, leading to a better description of the density low-range temperature dependence.

- the hypothesis that $\left.\frac{\partial h}{\partial p}\right)_{T}=0$ is lifted, with $\left.\frac{\partial h}{\partial p}\right)_{T}=b$ in the NASG framework.

- the SG sound speed $c^{2}=\gamma . r . T$, implies $\left.\frac{\partial c}{\partial T}\right)_{p}>0$, whereas the opposite is observed for most liquids. The NASG framework corrects that behavior, since

$$
c^{2}=\gamma \cdot r \cdot T\left(1+b \cdot \frac{\left(p+p_{\infty}\right)}{r \cdot T}\right)^{2},
$$

with the second term in the parenthesis becoming dominant at low temperatures. 

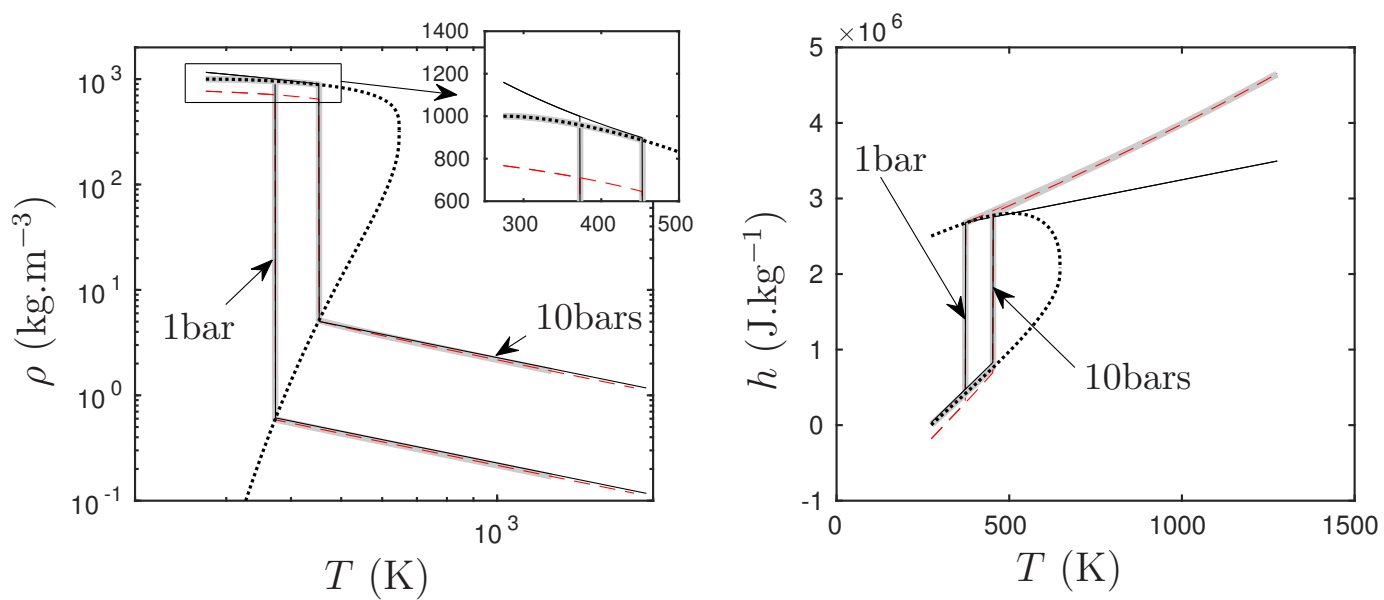

Figure 1: $\mathrm{H}_{2} \mathrm{O}$ density and enthalpy as functions of the temperature, at $p=1$ and 10 bars. Included (thick light gray) are the NIST reference [13], the SRK EOS (dashed, red), and the NASG EOS [12] (plain). The dotted curve corresponds to the saturation curves.

Despite these advantages, significant errors in the gas heat capacity were obtained, as hinted by the enthalpy plots of Fig. 1, water heat capacities $C_{p}$ reported in [12] are 903 or $1401 \mathrm{~J} / \mathrm{kg} / \mathrm{K}$ depending on the temperature range, very far from its actual value of $1996 \mathrm{~J} / \mathrm{kg} / \mathrm{K}$. Similar errors were obtained in the case of dioxygen $\mathrm{O}_{2}$, as later shown in Tab. 1.

This error comes from the method [12], in which the parameters are computed as to fit the reference saturation curves. Fig. 1.b illustrates well the error source: the saturation curve is not tangent to the isobaric properties.

\section{Proposed method for obtaining the NASG EOS parameters}

This Section presents a new method for setting the NASG EOS parameters. Instead of fitting the parameters as to match the saturation properties as in [12], the idea is to loosen the precision at saturation, and instead focus on obtaining satisfying behavior over a wide range of temperature and pressure. In particular, equality between the liquid and gas phase chemical 
potentials

$$
g=\left(\gamma C_{v}-q^{\prime}\right) T-C_{v} T \ln \frac{T^{\gamma}}{\left(p+p_{\infty}\right)^{\gamma-1}}+b p+q
$$

at the saturation curve $p=p_{\text {sat }}(T)$ will not be automatically satisfied. Reciprocally, this implies that the saturation pressure relation $p=p_{\text {sat }}(T)$ will not be obtainable from the EOS parameters. The implications of relaxing that constraint are discussed in Sec. 3.4. Under that assumption, we can then set the coefficients for each phase independently, significantly simplifying the procedure.

Within the NASG framework, the specific volume $v=1 / \rho$, mass enthalpy $h$, internal energy $e$ and sound velocity $c$ read respectively

$$
\left\{\begin{aligned}
v(p, T) & =\frac{\left(C_{p}-C_{v}\right) T}{p+p_{\infty}}+b \\
h(p, T) & =C_{p} T+b p+q \\
e(p, T) & =\frac{p+\gamma p_{\infty}}{p+p_{\infty}} C_{v} T+q, \\
c^{2}(p, v) & =\frac{\gamma v^{2}\left(p+p_{\infty}\right)}{v-b} .
\end{aligned}\right.
$$

where the following parameters are needed for each phase : $\gamma, p_{\infty}, C_{v}\left(\right.$ or $\left.C_{p}\right)$, $q$ and $b$. Let us now determine the EOS parameters for $\mathrm{O}_{2}$ as an example.

\subsection{Coefficients for the liquid phase}

From deriving Eq. (3), we can write:

$$
\left.\left.C_{p}=\frac{\partial h}{\partial T}\right)_{p}, \quad \text { and } \quad b=\frac{\partial h}{\partial p}\right)_{T} .
$$

These parameters can then readily be measured from fitting linearly the NIST reference mass enthalpy data: 
- the heat capacity $C_{p}$ is to be obtained from the enthalpy curve at a pressure $p_{0}$ far below the critical pressure as to exclude any real gas effect.

- similarly, the covolume $b$ is deduced from the enthalpy curve at a temperature $T_{0}$ sufficiently low (far from the critical temperature).

The procedure is illustrated in Fig. 2, for $\mathrm{O}_{2}$. The left plot indicates that
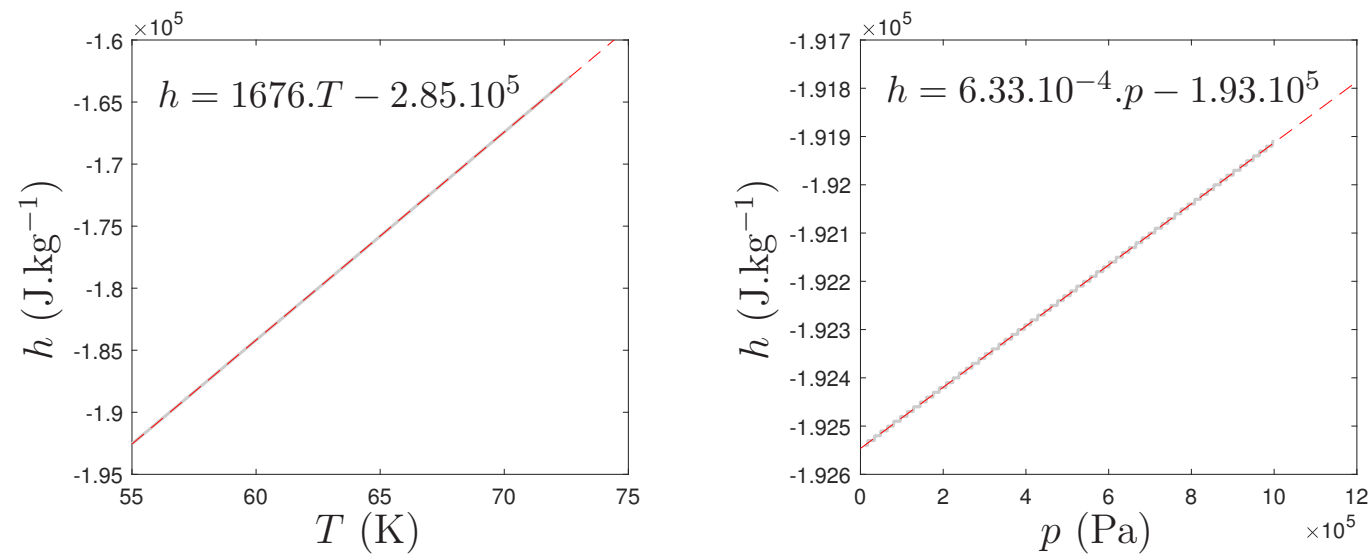

Figure 2: Evolution of $\mathrm{O}_{2}$ enthalpy with the temperature at the pressure 0.1bar (to the right) and with the pressure at the temperature $55 \mathrm{~K}$ (left) for the dioxygen with the NIST reference (solid grey cruves) and with the linear regressions (dashed red curves)

$C_{p}=1676 \mathrm{~J} \cdot \mathrm{kg}^{-1} \cdot \mathrm{K}^{-1}$, and the right plot shows that $b=6.335 \times 10^{-4} \mathrm{~m}^{3} \cdot \mathrm{kg}^{-1}$. The reference energy $q$ can then be obtained from either curve, by using the origin coordinate as:

$$
q=h_{f i t}\left(p=0, T_{0}\right)-C_{p} \cdot T_{0}=-1.926 \times 10^{5}-1676 \times 55=-284730 \mathrm{~J} \mathrm{~kg}^{-1}
$$

Note that using the other formula $h_{f i t}\left(p_{0}, T=0\right)-b . p_{0}$ leads to a close value $q=-284746 \mathrm{~J} \mathrm{~kg}^{-1}$, showing the accuracy of the procedure, as well as the relevance of the NASG thermodynamic description. 
Indeed, fitting the parameters on such a limited pressure and temperature ranges is voluntary, and does not preclude a much larger validity, as shown in the next Section. The idea is simply to choose the parameters based on reference data as far as possible from the saturation and/or the critical point, where the properties show almost non-linearity.

The only remaining parameters are now $\gamma$ (alternatively $C_{v}$ ) and $p_{\infty}$. The strategy is to set the parameters as to obtain asymptotically accurate thermodynamic properties at low temperatures. The specific volume and sound speed equations (3) may be rewritten as to obtain

$$
\gamma=\frac{c^{2}(v-b)^{2}}{v^{2} C_{p} T}+1
$$

which can be evaluated in conditions far from the critical point. At $T=55 \mathrm{~K}$ $\left(\approx T_{c} / 3\right)$, the NIST reference database gives, for $\mathrm{O}_{2}$

$$
v=7.670 \times 10^{-4} \mathrm{~m}^{3} \mathrm{~kg}^{-1}, \quad c=1.127 \times 10^{3} \mathrm{~ms}^{-1},
$$

leading to $\gamma=1.42 . p_{\infty}$ can then be obtained from rewriting (3), to give

$$
p_{\infty}=\frac{(\gamma-1) C_{p} T}{\gamma(v-b)}-p,
$$

to be evaluated under the same conditions, giving $p_{\infty}=2.034 \times 10^{8} \mathrm{~Pa}$.

\subsection{Coefficients for the vapor phase}

For the vapor phase, we assume $b$ and $p_{\infty}$ to be zero, reducing the system to the ideal gas equation of state with constant heat capacity. In that case, a single parameter is left to determine, as $C_{p}$ and $C_{v}$ relate through Mayer's relation $C_{p}-C_{v}=r$, where $r=R / W, R$ being the universal gas constant and $W$ the gas molar mass. For instance, we can choose $C_{v}$ as to fit the low temperature range, as illustrated in the left plot of Fig. 3, leading to $C_{v}=6.500 \times 10^{2} \mathrm{~J} \mathrm{~kg}^{-1} \mathrm{~K}^{-1}$. 

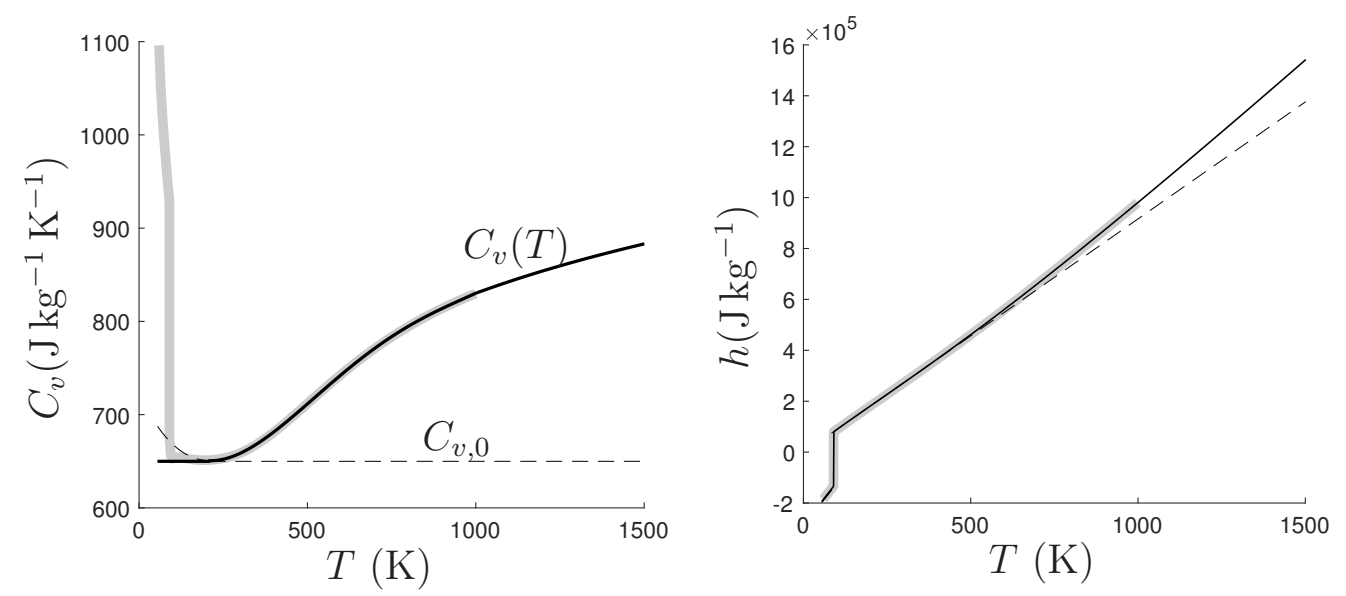

Figure 3: Dioxygen isochoric heat capacity and mass enthalpy dependence with temperature at 1 bar. Thick light gray: NIST reference. Dashed lines: NASA coefficients (for $T<208 \mathrm{~K}$ ), and constant $C_{v}$ as obtained Sec. 3.2. Plain line: $C_{v}(T)$ as obtained following 4.

\subsection{Results}

The parameters obtained by following the above step-by-step procedure are summarized in Tab. 1, which also reports the previous set of parameters obtained in [12]. Note that the heat capacity ratio and heat capacity $C_{p}$ have now satisfactory values, much closer to the naive expectation of $\gamma=1.4$ for a diatomic gas.

The corresponding density and enthalpy temperature dependencies are reported in Fig. 4, showing excellent agreement over the whole temperature range, given the pressure remains sufficiently below the critical pressure $p_{c}$, or approximately $p<p_{c} / 3$. Note that no thermochemical equilibrium is used here: the use of either phase parameters (liquid or gas) is solely decided according to the sign of $T-T_{\text {sat }}$. Accurate solvers for the thermo-chemical equilibrium are available within this context [14, 15].

The procedure was repeated for $\mathrm{H}_{2}, \mathrm{H}_{2} \mathrm{O}, \mathrm{N}_{2}$ and $\mathrm{C}_{12} \mathrm{H}_{26}$, for which the 


\begin{tabular}{|l|c|c|c|c|}
\cline { 2 - 5 } \multicolumn{1}{c|}{} & $\mathrm{O}_{2(l)}$ & $\mathrm{O}_{2(g)}$ & $\mathrm{O}_{2(l)}[12]$ & $\mathrm{O}_{2(g)}[12]$ \\
\hline$C_{p}\left(\mathrm{~J} \mathrm{~kg}^{-1} \mathrm{~K}^{-1}\right)$ & $1.676 \times 10^{3}$ & $9.194 \times 10^{2}$ & $1.741 \times 10^{3}$ & $5.520 \times 10^{2}$ \\
\hline$\gamma$ & 1.419 & 1.414 & 2.200 & 1.850 \\
\hline$b\left(\mathrm{~m}^{3} \mathrm{~kg}^{-1}\right)$ & $6.335 \times 10^{-4}$ & 0 & $4.570 \times 10^{-4}$ & 0 \\
\hline$p_{\infty}(\mathrm{Pa})$ & $2.034 \times 10^{8}$ & 0 & $2.036 \times 10^{8}$ & 0 \\
\hline$q\left(\mathrm{~J} \mathrm{~kg}^{-1}\right)$ & $-2.847 \times 10^{5}$ & $-2.104 \times 10^{3}$ & $-2.902 \times 10^{5}$ & $2.927 \times 10^{4}$ \\
\hline
\end{tabular}

Table 1: NASG parameters in SI units, as obtained following the procedure presented in Sec. 3 at the left and following [12] at the right, for dioxygen.
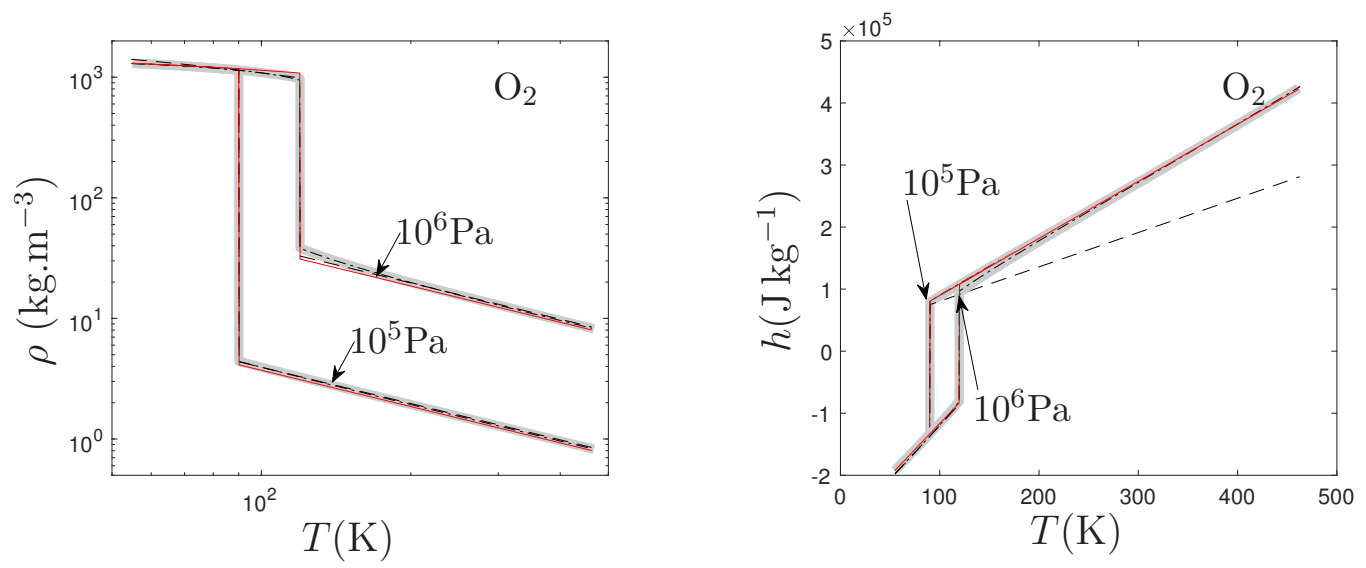

Figure 4: Dioxygen density and mass enthalpy temperature dependencies as obtained with the new set of parameters (red plain line) at $p=10^{5} \mathrm{~Pa}$ and $p=10^{6} \mathrm{~Pa}$. The light-gray heavy line reproduces the NIST reference, the dotted-dashed curve the SRK EOS, and the dashed curves the NASG, with the previous method [12]. 
parameters are reported in Tab. 2. Results for density and enthalpy as functions of temperature at $10^{5} \mathrm{~Pa}$ and $10^{6} \mathrm{~Pa}$ are included in Fig. 5 .

\begin{tabular}{|l|c|c|}
\cline { 2 - 3 } \multicolumn{1}{c|}{} & $\mathrm{H}_{2(l)}$ & $\mathrm{H}_{2(g)}$ \\
\hline$C_{p}$ & $7.100 \times 10^{3}$ & $1.040 \times 10^{4}$ \\
\hline$\gamma$ & 1.358 & 1.681 \\
\hline$b$ & $1.106 \times 10^{-2}$ & 0 \\
\hline$p_{\infty}$ & $1.355 \times 10^{7}$ & 0 \\
\hline$q$ & $-1.531 \times 10^{5}$ & $2.543 \times 10^{5}$ \\
\hline
\end{tabular}

\begin{tabular}{|l|c|c|}
\cline { 2 - 3 } \multicolumn{1}{c|}{} & $\mathrm{H}_{2} \mathrm{O}_{(l)}$ & $\mathrm{H}_{2} \mathrm{O}_{(g)}$ \\
\hline$C_{p}$ & $4.185 \times 10^{3}$ & $1.908 \times 10^{3}$ \\
\hline$\gamma$ & 1.012 & 1.328 \\
\hline$b$ & $9.203 \times 10^{-4}$ & 0 \\
\hline$p_{\infty}$ & $1.835 \times 10^{8}$ & 0 \\
\hline$q$ & $-1.143 \times 10^{6}$ & $1.957 \times 10^{6}$ \\
\hline
\end{tabular}

\begin{tabular}{|l|c|c|||c|c|c|}
\cline { 3 - 6 } \multicolumn{1}{c|}{} & $\mathrm{N}_{2(l)}$ & $\mathrm{N}_{2(g)}$ & & $\mathrm{C}_{12} \mathrm{H}_{26(l)}$ & $\mathrm{C}_{12} \mathrm{H}_{26(g)}$ \\
\hline$C_{p}$ & $2.021 \times 10^{3}$ & $1.045 \times 10^{3}$ & $C_{p}$ & $2.356 \times 10^{3}$ & $2.688 \times 10^{3}$ \\
\hline$\gamma$ & 1.674 & 1.407 & $\gamma$ & 1.220 & 1.020 \\
\hline$b$ & $8.054 \times 10^{-4}$ & 0 & $b$ & $9.600 \times 10^{-4}$ & 0 \\
\hline$p_{\infty}$ & $1.476 \times 10^{8}$ & 0 & $p_{\infty}$ & $3.325 \times 10^{8}$ & 0 \\
\hline$q$ & $-2.784 \times 10^{5}$ & $-2.355 \times 10^{3}$ & $q$ & $-1.189 \times 10^{6}$ & $-1.049 \times 10^{6}$ \\
\hline
\end{tabular}

Table 2: Parameters in SI units, as obtained following the procedure presented Sec. 3, for dihydrogen, water, nitrogen and dodecane.

Density and enthalpy are better described in the liquid phase with the NASG EOS than with the SRK EOS (especially for $\mathrm{H}_{2}$ and $\mathrm{H}_{2} \mathrm{O}$ ). Dark lines in the $\mathrm{H}_{2} \mathrm{O}$ plot indicate the results as obtained with the parameters derived in [12], showing a significant improvement of the enthalpy prediction in both phases. The density is also better predicted in the liquid phase.

Indeed, as indicated by the $\mathrm{H}_{2}$ plots of Fig. 5, results get worse as the pressure get closer to the critical pressure (especially low for $\mathrm{H}_{2}: p_{c}=$ $1.300 \times 10^{6} \mathrm{~Pa}$ ). Yet, density and enthalpy predictions in the limits far from saturation are better than with the SRK EOS. That case aside, the other significant departure from the NIST reference concerns $\mathrm{H}_{2} \mathrm{O}$ at high temperature (from $800 \mathrm{~K}$ and above). This error can be suppressed, however, accounting for the variable $C_{p}$ as shown in Sec. 4. 

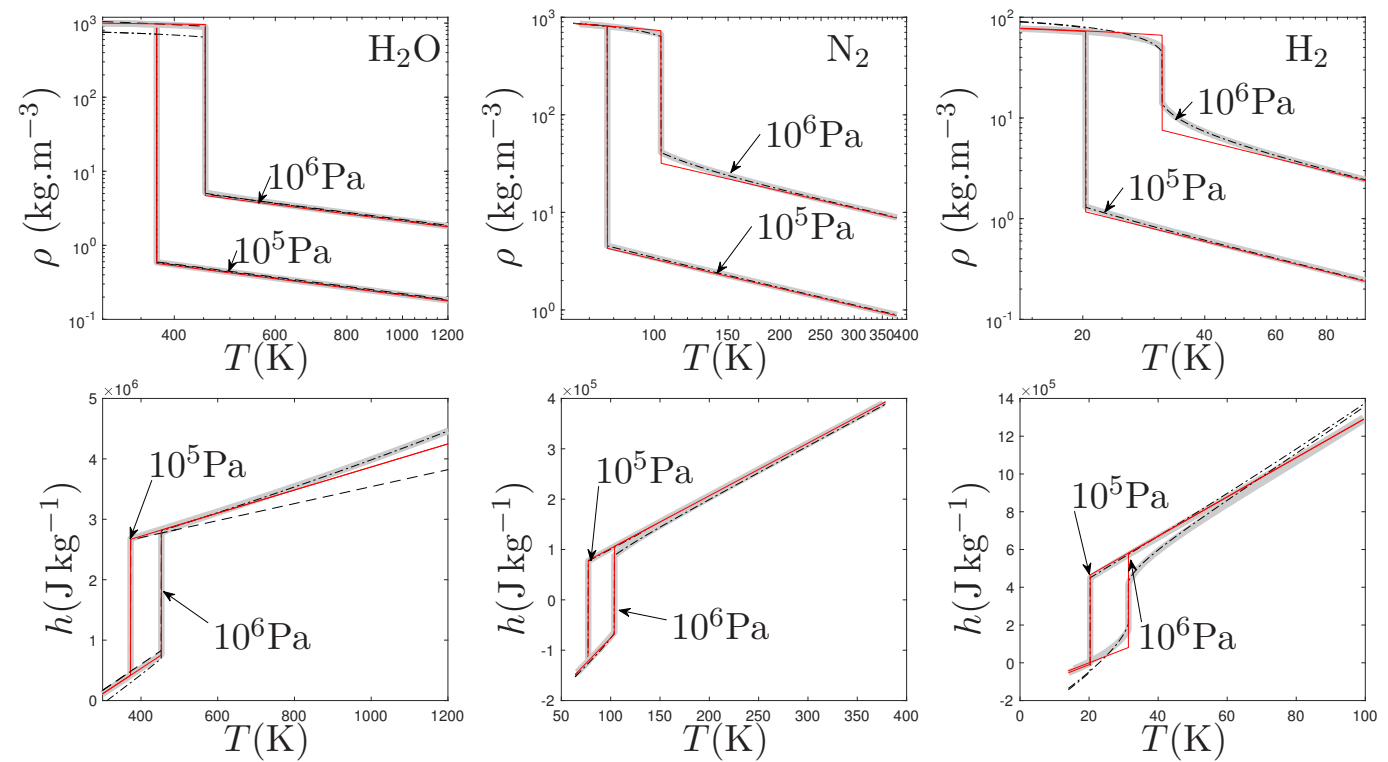

Figure 5: Validation of the NASG parameters: density and enthalpy temperature dependences at $10^{5} \mathrm{~Pa}$ and $10^{6} \mathrm{~Pa}$ as obtained with the parameters from Tab. 2. Dashed lines report results from [12] when available.

\subsection{Implications of not using the saturation curves in the proposed method}

Even though the saturation curves are not used in fitting the parameters as recommended in [12], agreement on saturation properties remains excellent in the sub-critical range of pressure. This is illustrated in Fig 6 , comparing the saturation pressure and latent heat dependencies with temperature as obtained with the new set of parameters with the NIST reference. Since the chemical potentials equality is not strictly enforced at saturation, the use of an analytic approximation for the saturation pressure is recommended for phase transfers computations such as [14, 15], which remain fully compatible. Figure [6] shows that use of Antoine's equation with appropriate coefficients [16] leads to a saturation pressure indistinguishable from the reference, whereas slight departures are obtained with the NASG formulation 
at high temperatures. For this Figure, we used the coefficients recommended on the NIST website [13] to approximate the saturation pressure (Pa.) as a function of $\mathrm{T}(\mathrm{K})$ as

$$
p_{\text {sat }}(T)=10^{A-\frac{B}{C+T}},
$$

with $A=3.9523, B=340.024$ and $C=-4.144$.
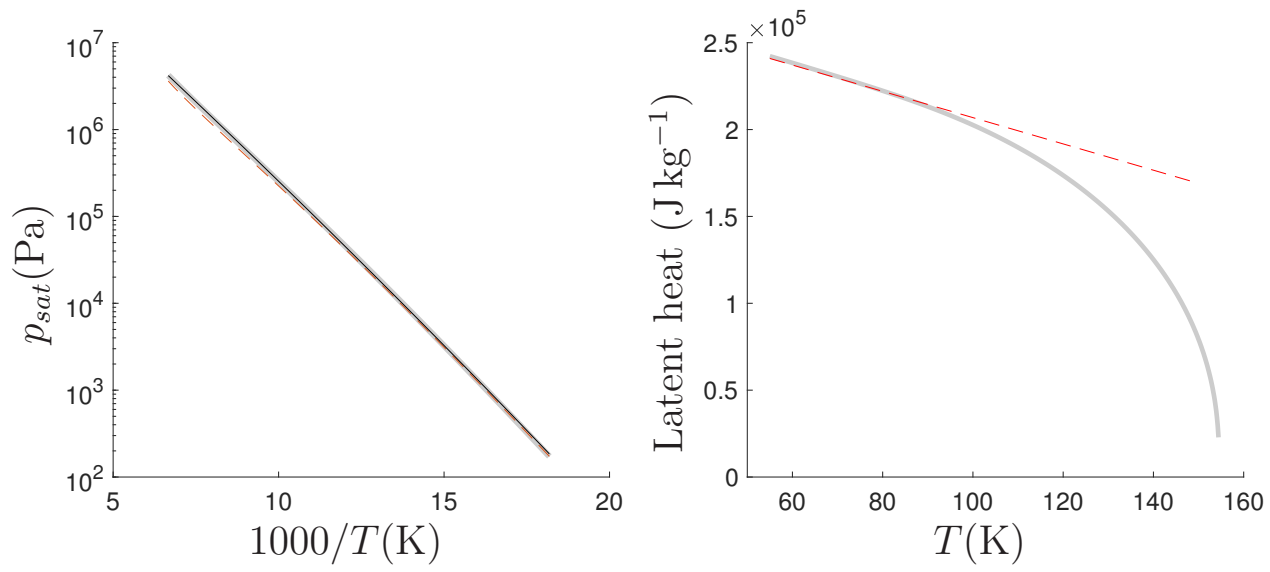

Figure 6: Dioxygen saturation pressure (left) and latent heat (right) dependence with temperature. The light-gray heavy lines reproduce the NIST reference, and the red-dashed curve correspond to the NASG values obtained with updated parameters from Table 1 The solid line in the left plot, indistinguishable from the NIST reference corresponds to the saturation pressure as obtained with Antoine's equation [16].

In addition from providing a wider validity range, our approach to compute the NASG parameters also allows significant cpu cost reduction: The obtention of $p_{\text {sat }}$ following the initial proposal [12] requires a root-finding procedure, whereas it is here replaced by an analytical expression (8).

\section{NASG extension to variable heat capacity}

In configurations where the temperature varies significantly (e.g. in reactive flows), the constant heat capacity approximation fails, as is clearly 
visible in the $\mathrm{H}_{2} \mathrm{O}$ mass enthalpy plot of Fig. 5. It is therefore worth investigating whether this assumption can be relaxed within the NASG framework. Let us now, as is rather classical for reactive flows, consider a heat capacity $C_{p}$ dependent on the temperature

$$
\left.\frac{\partial h}{\partial T}\right)_{p}=C_{p}(T),
$$

which, through integration, leads to

$$
h(p, T)=\int C_{p}(T) d T+f(p) .
$$

Assuming a linear profile of the function $f(p)=b \cdot p+q$ with constant covolume $b$ and reference energy $q$ (as in [12]), we have

$$
\left.\frac{\partial h}{\partial p}\right)_{T}=f^{\prime}(p)=b,
$$

which, combined with the third Maxwell's relation

$$
\left.\left.\frac{\partial h}{\partial p}\right)_{T}=v-T \frac{\partial v}{\partial T}\right)_{p}
$$

yields

$$
\left.v-b=T \frac{\partial v}{\partial T}\right)_{p}
$$

and eventually

$$
\left\{\begin{array}{l}
h(p, T)=\int C_{p}(T) d T+b \cdot p+q, \\
v(p, T)=g(p) \cdot T+b,
\end{array}\right.
$$

where $g(p)$ is the function of $p$ arising from integration of (13), shown in AppendixA to be of the form

$$
g(p)=\frac{r}{p+p_{\infty}}
$$


with $r=C_{p}(T)-C_{v}(T)$, and $p_{\infty}$ being two constants.

Within this framework, the density, specific enthalpy and internal energy then read

$$
\left\{\begin{array}{l}
v(p, T)=\frac{r T}{p+p_{\infty}}+b \\
h(p, T)=\int C_{p}(T) d T+b p+q \\
e(p, T)=h(p, T)-p v(p, T)=\int C_{v}(T) d T+\frac{r T p_{\infty}}{p+p_{\infty}}+q
\end{array}\right.
$$

In addition,

$$
\left.\frac{\partial s}{\partial T}\right)_{p}=\frac{C_{p}(T)}{T}
$$

and (Maxwell's relation)

$$
\left.\left.\frac{\partial s}{\partial p}\right)_{T}=-\frac{\partial v}{\partial T}\right)_{p}
$$

which can be combined into

$$
s(p, T)=\int C_{p}(T) / T d T-r \ln \left(p+p_{\infty}\right)+q^{\prime}
$$

where $q^{\prime}$ is a constant.

From equations (16) and (19),

$$
d s=\frac{C_{v}(T)}{\left(p+p_{\infty}\right)} d p+\frac{C_{p}(T)}{(v-b)} d v
$$

finally leading to the speed of sound as

$$
\left.c^{2}=-v^{2} \frac{\partial p}{\partial v}\right)_{s}=\frac{C_{p}(T)}{C_{v}(T)} \frac{\left(p+p_{\infty}\right)}{(v-b)} v^{2}
$$

Finally, the density, specific enthalpy, internal energy, entropy, chemical 
potential and sound speed read

$$
\left\{\begin{aligned}
v(p, T) & =\frac{r T}{p+p_{\infty}}+b \\
h(p, T) & =\int C_{p}(T) d T+b p+q \\
e(p, T) & =\int C_{v}(T) d T+\frac{r T p_{\infty}}{p+p_{\infty}}+q \\
s(p, T) & =\int C_{p}(T) / T d T-r \ln \left(p+p_{\infty}\right)+q^{\prime} \\
g(p, T) & =h(p, T)-T s(p, T) \\
& =\int C_{p}(T) d T-T \int C_{p}(T) / T d T-T q^{\prime}+r T \ln \left(p+p_{\infty}\right)+b p+q \\
c^{2}(p, T) & =\frac{C_{p}(T)}{C_{v}(T)} r T\left(1+b \frac{\left(p+p_{\infty}\right)}{r T}\right)^{2}
\end{aligned}\right.
$$

The Noble-Abel Stiffened gas equation of state defined as above can then tackle variable heat capacity. In particular, for reactive flows, implementing the NASA polynomials is now straight-forward for example. As illustrated in Fig. 3 for $\mathrm{O}_{2}$, and anticipated in the previous Section, the possibility to account for variable $C_{p}$ fully corrects the errors at high temperature observed in Fig. 5 .

Appended with variable $C_{p}$ formulation, it is shown in AppendixB that

$$
\left.\left.\frac{\partial^{2} e}{\partial s^{2}}\right)_{v} \frac{\partial^{2} e}{\partial v^{2}}\right)_{s}-\left(\frac{\partial^{2} e}{\partial v \partial s}\right)^{2}=\frac{r T^{2}}{\left(C_{p}(T)-r\right)(v-b)^{2}}>0
$$

confirming that the resulting EOS remains convex regardless of the fit retained for $C_{p}(T)$.

\section{Practical implementation: a thermodynamic closure for the sim- ulation of multiphase reactive flows}

The NASG equation of state, with updated coefficients, can directly be used in multiphase flow solver based upon Kapila's 7-equation model [17], or 
any solver using separate EOS for each phase [3]. When the flow is assumed to be in thermal and mechanical equilibrium locally [18], the derivation of relations relating the mixture conservative variables $(v, e)$ with the intensive quantities $(p, T)$ becomes necessary. In particular, since computation of the pressure has to be performed at each grid point every time-step, an explicit formulation for $p$ is of high interest, as it is typically much faster than a root-finding procedure.

This Section provides explicit analytic thermodynamic closure for a mixture consisting of a liquid phase and a multi-component gas phase.

\subsection{Constant heat capacity}

Let us remind the formulation obtained in the case of constant heat capacity obtained in [15]. The given mixture described by a mass fraction distribution $Y_{k}$, where $k$ identifies the $k^{\text {th }}$ component of the mixture. In our approach, the $Y_{k}$ are mass fractions within a control volume, which contains both the liquid and gas phases. In particular, we identify the following components:

- $k=1$ for the considered liquid (fuel, oxidizer, water, depending on the application),

- $k=2$ for the gas component corresponding to vapor of species 1 ,

- $k=3, \ldots, N$ for the remaining gas components, considered non-condensable.

Using the notations introduced above, one can obtain $(v, e)$ from $(p, T)$ 
through

$$
\left\{\begin{array}{l}
v=\sum_{k=1}^{N} Y_{k} v_{k}(p, T) \\
e=\sum_{k=1}^{N} Y_{k} e_{k}(p, T)
\end{array}\right.
$$

where the $v_{k}$ and $e_{k}$ are calculated with (3), with use of the coefficients for the $k^{\text {th }}$ component.

Inverting System 24 to obtain $(p, T)$ from $(v, e)$ is less straight-forward. It leads to a second-order polynomial equation with a single positive root [15] for $p$ :

$$
p(v, e)=\frac{b+\sqrt{b^{2}+4 a c}}{2 a}
$$

with

$$
\left\{\begin{aligned}
a & =\bar{C}_{v} \\
b & =\left(\frac{e-\bar{q}}{v-\bar{b}}\right)\left(\bar{C}_{p}-\bar{C}_{v}\right)-p_{\infty, 1} \bar{C}_{v}-p_{\infty, 1} Y_{1}\left(C_{p, 1}-C_{v, 1}\right), \\
c & =\left(\frac{e-\bar{q}}{v-\bar{b}}\right) p_{\infty, 1}\left[\bar{C}_{p}-\bar{C}_{v}-Y_{1}\left(C_{p, 1}-C_{v, 1}\right)\right]
\end{aligned}\right.
$$

where mixture quantities are introduced:

$$
\bar{C}_{v}=\sum_{k=1}^{N} Y_{k} C_{v, k}, \quad \bar{C}_{p}=\sum_{k=1}^{N} Y_{k} C_{p, k}, \quad \bar{q}=\sum_{k=1}^{N} Y_{k} q_{k}, \quad \bar{b}=\sum_{k=1}^{N} Y_{k} b_{k} .
$$

Once the pressure is known, the temperature is easily obtained as

$$
T=(v-\bar{b})\left(\sum_{k=1}^{N} \frac{Y_{k}\left(C_{p, k}-C_{v, k}\right)}{p+p_{\infty, k}}\right)^{-1}
$$

We refer the reader to [14, 15, 19] for computational examples with the above thermodynamic closure. 


\subsection{Variable heat capacity}

When considering variable heat capacity, obtaining $(v, e)$ from the intensive variables $(p, T)$ remains possible through equation (24), with use of updated $v_{k}(p, T)$ and $e_{k}(p, T)$ expressions (22).

However, because of the $\int C_{v} d T$ term in the energy expression, it is no longer possible to obtain explicit expressions for $(p, T)$ from $(v, e)$. The brute force method consists in writing an iterative solver for $p$, and deducing $T$ from (28) (or, alternatively, iteratively solving for $T$ and analytically obtain $p$ ).

It has to be noted, however, that solving for $p$ is typically done at every point and time step of a multiphase flow numerical simulation so efficiency in its computation is of paramount importance.

A faster and more efficient method can be obtained by noting that the constant heat capacity assumption only fails at high temperatures, where the liquid is no longer present. We then suggest to define the $C_{p}(T)$ function as a continuous $C^{0}$ function (piece-wise $C^{\infty}$ ) with:

$$
\begin{cases}C_{p}(T)=C_{p}^{v a r}(T) & \text { if } T>T_{0} \\ C_{p}(T)=C_{p, 0} & \text { if } T \leq T_{0},\end{cases}
$$

where $C_{p}^{v a r}(T)$ can be any function (e.g. a NASA polynomial), and $C_{p, 0}$ is that found in Sec. 3. $T_{0}$ simply has to be calculated such as $C_{p}^{v a r}\left(T_{0}\right)=C_{p, 0}$ which, in the case of $\mathrm{O}_{2}$ leads to $T_{0}=208 K$ with the parameters from Tab. 2 (577K for water). That temperature is high enough to ensure that the flow is mono-phase in the variable $C_{p}$ domain. If the gas phase consists of various gas components, each should be given the same $T_{0}$ as to maintain the validity of (25). The method is illustrated in Fig. 2, in which was added the $C_{v}(T)$ and $h(T)$ curves following the formalism of Eq. (29). 
The obvious advantage of this method is that the multi-phase flow thermodynamic closure for the pressure (25) remains valid and explicit, and, most importantly, remains unique in the sense that the same formulation is used everywhere in the multiphase flow. Also, the simple explicit phase transition solvers presented previously [14, 15] remain valid.

Note that this method is also applicable on the liquid side, where a variable $C_{p}$ could be implemented below a certain temperature, should the application require it. In the unlikely event where a constant $C_{p}$ is not a viable assumption on either side of saturation, the present work can still serve with the implementation of an iterative solver for the pressure.

\section{Conclusion}

A new method for setting the parameters in the Noble-Abel StiffenedGas equation of state has been presented. Instead of focusing on fitting the thermodynamic properties at saturation, the proposed method considers the properties of the liquid and gas state entirely independently. As a result, large ranges of validity are obtained, typically from freezing point to way above saturation (e.g. $\mathrm{H}_{2} \mathrm{O}: 273-800 \mathrm{~K}, \mathrm{O}_{2}$ : 55-600K,...). Saturation properties predictions are shown to remain excellent with the new method, given the pressure remains sub-critical.

To further expand the temperature validity range up to combustion temperatures, this work also extends the Noble-Abel Stiffened gas equation of state as to encompass variable heat capacities, while preserving its convexity. Through consideration of NASA polynomials for the gas phase [13], the upper temperature limit is effectively lifted, making the thermodynamic closure valid for combustion applications that include a liquid phase. 
The explicit thermodynamic closure previously derived [15] has been updated accordingly, as to include these novelties, providing a suitable thermodynamic closure for the simulation of multiphase reactive flows.

\section{Acknowledgements}

École Centrale Marseille is gratefully acknowledged for supporting MarieAstrid's research internship at M2P2. We also acknowledge support from Labex MEC (ANR-10-LABX-0092) and the A*MIDEX project (ANR-11IDEX-0001-02), funded by the "Investissements d'Avenir".

\section{AppendixA. Variable $C_{p}$ NASG equation of state derivation}

This Section provides the proof required to fill the gap between Eqs. (14) and (22). Starting over from (14), the internal energy reads, by definition,

$$
e(p, T)=h(p, T)-p \cdot v(p, T)=\int C_{p}(T) d T+q-p T g(p)
$$

Combining this expression for the internal energy with the differential form

$$
\left.\left.d e=\frac{\partial e}{\partial p}\right)_{T} d p+\frac{\partial e}{\partial T}\right)_{p} d T
$$

or, alternatively

$$
\left.\left.\left.\left.\frac{\partial e}{\partial T}\right)_{v}=C_{v}(T)=\frac{\partial e}{\partial p}\right)_{T} \frac{\partial p}{\partial T}\right)_{v}+\frac{\partial e}{\partial T}\right)_{p}
$$

yields

$$
\left\{\begin{array}{l}
\left.\frac{\partial e}{\partial T}\right)_{p}=C_{p}(T)-p \cdot g(p) \\
\left.\frac{\partial e}{\partial p}\right)_{T}=-T\left(g(p)+p \cdot g^{\prime}(p)\right)
\end{array}\right.
$$


Reformulating (14) as

$$
\left.\left.\frac{\partial p}{\partial T}\right)_{v}=\left(\frac{\partial T}{\partial p}\right)_{v}\right)^{-1}=\frac{-g(p)}{g^{\prime}(p) T}
$$

Equation (A.3) leads to

$$
C_{v}(T)=C_{p}(T)+\frac{g(p)^{2}}{g^{\prime}(p)} .
$$

$C_{v}$ and $C_{p}$ depending only on temperature,

$$
C_{v}(T)-C_{p}(T)=\frac{g(p)^{2}}{g^{\prime}(p)}=-r,
$$

where $r$ is a constant. Integration of (A.7) leads to :

$$
g(p)=\frac{r}{(p+r C)}
$$

where $C$ is another constant.

Maxwell's second relation implies

$$
\left.\left.\frac{\partial e}{\partial v}\right)_{T}=T \frac{\partial p}{\partial T}\right)_{v}-p
$$

which, combined with Eqs. (A.5) and (A.8) yields

$$
\left.\frac{\partial e}{\partial v}\right)_{T}=\frac{-g(p)}{g^{\prime}(p)}-p=r C
$$

and, through integration

$$
e(v, T)=C r v+f(T)
$$

where $f(T)$ remains to be determined.

With the help of (A.3),

$$
\left.\frac{\partial e}{\partial T}\right)_{v}=C_{v}(T)=C_{p}(T)-r=f^{\prime}(T)
$$


allowing the determination of function $f$ as

$$
f(T)=\int C_{p}(T) d T-r T+D
$$

with $D$ being yet another integration constant. Eq. (A.11) now provides an expression for the internal energy in function of specific volume and temperature

$$
e(v, T)=C r v+\int C_{p}(T) d T-r T+D
$$

which, combined with (14) leads to

$$
e(p, T)=\int C_{p}(T) d T-\frac{r T p}{(p+r C)}+D+r C b .
$$

Eventually, comparing Eqs. (A.1) and (A.15) relates the integration constants as

$$
D=q-r C b
$$

Summarising now the equations obtained so far as

$$
\left\{\begin{array}{l}
v(p, T)=\frac{r T}{(p+r c)}+b \\
e(p, T)=\int C_{p}(T) d T+q-\frac{r T p}{(p+r C)} \\
e(v, T)=\int C_{p}(T) d T+q-r T+r C(v-b)
\end{array}\right.
$$

setting $C=\frac{p_{\infty}}{r}$, by analogy with the NASG EOS [12] eventually leads to the intended system of equations (22) given in Section 4 .

\section{AppendixB. Convexity of the NASG EOS including variable $C_{p}$}

Convexity is examined in this part and the state function $e(v, s)$ is needed. This one is not explicit but the following derivatives are available :

$$
\left\{\begin{array}{l}
\left.\frac{\partial e}{\partial s}\right)_{v}=\frac{\left.\frac{\partial e}{\partial T}\right)_{v}}{\left.\frac{\partial s}{\partial T}\right)_{v}} \\
\left.\left.\left.\left.\frac{\partial e}{\partial v}\right)_{s}=\frac{\partial e}{\partial v}\right)_{T}-\frac{\partial s}{\partial v}\right)_{T} \frac{\partial e}{\partial s}\right)_{v}
\end{array},\right.
$$


From (22), the following relations are readily obtained:

$$
\left\{\begin{array}{l}
\left.\frac{\partial e}{\partial T}\right)_{v}=C_{v}(T) \\
\left.\frac{\partial e}{\partial v}\right)_{T}=p_{\infty} \\
\left.\frac{\partial s}{\partial v}\right)_{T}=\frac{r}{v-b} \\
\left.\frac{\partial s}{\partial T}\right)_{v}=\frac{C_{v}(T)}{T}
\end{array},\right.
$$

Then (B.1) becomes:

$$
\left\{\begin{array}{l}
\left.\frac{\partial e}{\partial s}\right)_{v}=T \\
\left.\frac{\partial e}{\partial v}\right)_{s}=-p
\end{array},\right.
$$

The second derivatives of $e(v, s)$ must now be computed with the help of the preceding relations:

$$
\begin{gathered}
\left.\left.\frac{\partial^{2} e}{\partial v^{2}}\right)_{s}=-\frac{\partial p}{\partial v}\right)_{s}=-\frac{\left.\frac{\partial p}{\partial T}\right)_{s}}{\left.\frac{\partial v}{\partial T}\right)_{s}}=\frac{C_{p}(T)}{C_{v}(T)} \frac{\left(p+p_{\infty}\right)}{(v-b)}>0 \\
\left.\left.\frac{\partial^{2} e}{\partial s^{2}}\right)_{v}=\frac{\partial T}{\partial s}\right)_{v}=\frac{1}{\left.\frac{\partial s}{\partial T}\right)_{v}}=\frac{T}{C_{v}(T)}>0 \\
\left\{\begin{array}{l}
\left.\frac{\partial^{2} e}{\partial v \partial s}=\frac{\partial T}{\partial v}\right)_{s}=\frac{-r T}{C_{v}(T)(v-b)} \\
\left.\frac{\partial^{2} e}{\partial s \partial v}=-\frac{\partial p}{\partial s}\right)_{v}=\frac{-r T}{C_{v}(T)(v-b)}=\frac{\partial^{2} e}{\partial v \partial s}
\end{array}\right.
\end{gathered}
$$

From (B.4), (B.5) and (B.6) the following term is unconditionally positive as:

$$
\left.\left.\frac{\partial^{2} e}{\partial s^{2}}\right)_{v} \frac{\partial^{2} e}{\partial v^{2}}\right)_{s}-\left(\frac{\partial^{2} e}{\partial v \partial s}\right)^{2}=\frac{r T^{2}}{C_{v}(T)(v-b)^{2}}>0
$$

The equation of state (22) is thus convex whatever the polynomial profiles of $C_{p}(t)$, or alternately $C_{v}(T)$. 


\section{References}

[1] F. H. Harlow, A. A. Amsden, A numerical fluid dynamics calculation method for all flow speeds, Journal of Computational Physics 8 (2) (1971) 197-213.

[2] H. Lund, A hierarchy of relaxation models for two-phase flow, SIAM Journal on Applied Mathematics 72 (6) (2012) 1713-1741.

[3] M. G. Rodio, P. M. Congedo, R. Abgrall, Two-phase flow numerical simulation with real-gas effects and occurrence of rarefaction shock waves, European Journal of Mechanics B/Fluids 45 (2014) 20-35.

[4] T. Schmitt, Y. Méry, M. Boileau, S. Candel, Large-eddy simulation of oxygen/methane flames under transcritical conditions, Proceedings of the Combustion Institute 33 (1) (2011) 1383-1390.

[5] P. Gaillard, V. Giovangigli, L. Matuszewski, A diffuse interface lox/hydrogen transcritical flame model, Combustion Theory and Modelling 20 (3) (2016) 486-520.

[6] C. Laurent, L. Esclapez, D. Maestro, G. Staffelbach, B. Cuenot, L. Selle, T. Schmitt, F. Duchaine, T. Poinsot, Flame-wall interaction effects on the flame root stabilization mechanisms of a doubly-transcritical lo 2/lch 4 cryogenic flame, Proceedings of the Combustion Institute.

[7] J. D. Van der Waals, Over de Continuiteit van den Gas-en Vloeistoftoestand, Vol. 1, Sijthoff, 1873. 
[8] O. Redlich, J. N. Kwong, On the thermodynamics of solutions. v. an equation of state. fugacities of gaseous solutions., Chemical reviews 44 (1) (1949) 233-244.

[9] G. Soave, Equilibrium constants from a modified redlich-kwong equation of state, Chemical Engineering Science 27 (6) (1972) 1197-1203.

[10] D.-Y. Peng, D. B. Robinson, A new two-constant equation of state, Industrial \& Engineering Chemistry Fundamentals 15 (1) (1976) 59-64.

[11] O. Le Métayer, J. Massoni, R. Saurel, Elaboration des lois d'état d'un liquide et de sa vapeur pour les modèles d'écoulements diphasiques, International journal of thermal sciences 43 (3) (2004) 265-276.

[12] O. Le Métayer, R. Saurel, The noble-abel stiffened-gas equation of state, Physics of Fluids (1994-present) 28 (4) (2016) 046102.

[13] P. J. Linstrom, W. Mallard, Nist chemistry webbook; nist standard reference database no. 69 .

[14] A. Chiapolino, P. Boivin, R. Saurel, A simple phase transition relaxation solver for liquid-vapor flows, International Journal for Numerical Methods in Fluids 83 (7) (2017) 583-605.

[15] A. Chiapolino, P. Boivin, R. Saurel, A simple and fast phase transition relaxation solver for compressible multicomponent two-phase flows, Computers \& Fluids 150 (2017) 31-45.

[16] G. T. Brower, G. Thodos, Vapor pressures of liquid oxygen between the 
triple point and critical point, Journal of Chemical \& Engineering Data 13 (2) (1968) 262-264.

[17] A. Kapila, R. Menikoff, J. Bdzil, S. Son, D. Stewart, Two-phase modeling of deflagration-to-detonation transition in granular materials: Reduced equations, Physics of Fluids (1994-present) 13 (10) (2001) 30023024 .

[18] S. Le Martelot, R. Saurel, B. Nkonga, Towards the direct numerical simulation of nucleate boiling flows, International Journal of Multiphase Flow 66 (2014) 62-78.

[19] R. Saurel, P. Boivin, O. Le Métayer, A general formulation for cavitating, boiling and evaporating flows, Computers \& Fluids 128 (2016) $53-64$. 